\title{
Suppression of pulse splitting in two-core optical fibers with Kerr nonlinearity
}

\author{
Jin Hua Li, ${ }^{1}$ Kin Seng Chiang, ${ }^{2, *}$ and Kwok Wing Chow ${ }^{1,3}$ \\ ${ }^{1}$ Department of Mechanical Engineering, University of Hong Kong, Hong Kong SAR, China \\ ${ }^{2}$ Department of Electronic Engineering, City University of Hong Kong, Hong Kong SAR, China \\ ${ }^{3}$ e-mail: kwchow@hku.hk \\ ${ }^{*}$ Corresponding author: eeksc@cityu.edu.hk
}

Received October 19, 2012; accepted December 24, 2012;

posted January 7, 2013 (Doc. ID 178201); published January 29, 2013

\begin{abstract}
Intermodal dispersion (IMD) in a two-core fiber can cause significant pulse distortion or even pulse splitting. In this paper, by solving the generalized coupled nonlinear Schrödinger equations, we study the possibility of suppressing the effects of IMD with Kerr nonlinearity in a two-core fiber. We discover that, in the anomalous dispersion regime, when the IMD is sufficiently small and the linear coupling between the two cores is sufficiently strong, an input pulse power larger than the soliton power can prevent the pulse from broadening and splitting. The maximum IMD that can be balanced by nonlinearity increases with the input power. Our study shows that a proper balance of the group-velocity dispersion, the IMD, and the nonlinearity in a two-core fiber can maintain the integrity of the pulse over a long distance. (c) 2013 Optical Society of America

OCIS codes: $\quad 060.2310,060.2340,060.5530,060.1810,190.4370$.
\end{abstract}

\section{INTRODUCTION}

The existence of solitons in a single-mode fiber [1,2] is one of the most fascinating discoveries in fiber optics. A soliton in its simplest form is a pulse that propagates along a fiber without changing its shape. It is the result of balancing the anomalous group-velocity dispersion (GVD) with the Kerr nonlinearity in the fiber. The use of soliton pulses for long-distance optical transmission has created tremendous interest, and the properties of various optical soliton systems for different applications have been investigated in great detail (see [3-5] and references therein). At the same time, there have been extensive studies of various techniques to manipulate solitons in fibers. Soliton switching in nonlinear two-core fibers, in particular, has attracted much attention [6-12]. The recent interest in the use of multicore fibers for increasing the transmission capacity of optical communication systems [13-15], together with the development of multicore photonic-crystal fibers for nonlinear applications [16,17], suggests that the prospect of using nonlinear multicore fibers for optical pulse processing may become more promising than ever. In this paper, we analyze pulse propagation in a nonlinear two-core fiber with the objective of identifying the conditions to maintain the integrity of the pulse in the presence of intermodal dispersion (IMD).

A two-core fiber consisting of two parallel identical single-mode cores is a bimodal structure, which supports a symmetric mode and an antisymmetric mode. In the continuous-wave case, the beating of the two modes gives rise to the well-known phenomenon of power exchange between the two cores, which forms the basis of many optical coupling devices. In the case of pulse propagation, because the two modes in general have different group velocities, the input pulse may broaden or even split after propagating a certain distance and thus lose its identity $[18,19]$. This effect, known as IMD [18], can jeopardize the function of the two-core fiber as an optical switch, though it has been applied to the generation of high-repetition-rate pulse trains [20]. In the presence of significant IMD, soliton pulses are subject to distortion and splitting, just like low-power linear pulses [21]. A recent analysis shows that IMD can indeed deteriorate the switching characteristics of ultrashort pulses in nonlinear multicore fibers [22]. Previous studies of soliton switching in nonlinear

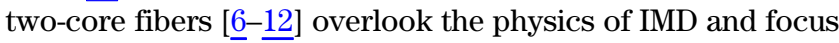
only on the balance of GVD and nonlinearity. In general, however, IMD exists in a two-core fiber [18]. It is of theoretical and practical interest to find out whether nonlinearity can balance both GVD and IMD in a two-core fiber to minimize pulse distortion and, in particular, to avoid pulse splitting.

There have been few studies on the interaction of IMD and nonlinearity in an optical fiber. Although the possibility of overcoming pulse broadening due to IMD by nonlinearity in a multimode fiber was predicted a long time ago [23-25], no detailed investigation on the propagation dynamics has ever been given. A numerical analysis of a birefringent fiber, which supports two polarized modes, shows the possibility of propagating soliton-like pulses in the fiber by balancing linear birefringence (actually polarization-mode dispersion) with nonlinearity [26]. In view of these early studies, it is reasonable to expect possible balance between IMD and nonlinearity in a two-core fiber. A two-core fiber, however, allows light to be transferred between two cores, which is phenomenally different from a multimode fiber or a birefringent fiber, where light is always confined in one core. It is yet to be confirmed whether, or to what extent, the IMD in a two-core fiber can be overcome by nonlinearity to avoid pulse splitting. In this paper, we address this issue by numerically solving the generalized coupled nonlinear Schrödinger equations. We discover that, in the anomalous dispersion regime, nonlinearity 
can suppress the effects of IMD, provided that the IMD is sufficiently small in comparison with the GVD and, at the same time, the linear coupling is sufficiently strong. We present propagation dynamics to elucidate these conditions.

\section{PULSE PROPAGATION IN A TWO-CORE FIBER}

We consider a lossless fiber that consists of two parallel identical single-mode cores. The composite two-core structure supports a symmetric mode and an antisymmetric mode (the supermodes) and their propagation along the fiber can be described by the following set of normalized coupled equations, which include the effects of Kerr nonlinearity [21]:

$$
\begin{aligned}
& i\left(\frac{\partial a_{+}}{\partial Z}+R_{1} \frac{\partial a_{+}}{\partial T}\right)+\frac{1}{2} \frac{\partial^{2} a_{+}}{\partial T^{2}}+\left|a_{+}\right|^{2} a_{+} \\
& \quad+2\left|a_{-}\right|^{2} a_{+}+a_{-}^{2} a_{+}^{*} \exp (-4 R Z)=0, \\
& i\left(\frac{\partial a_{-}}{\partial Z}-R_{1} \frac{\partial a_{-}}{\partial T}\right)+\frac{1}{2} \frac{\partial^{2} a_{-}}{\partial T^{2}}+\left|a_{-}\right|^{2} a_{-} \\
& \quad+2\left|a_{+}\right|^{2} a_{-}+a_{+}^{2} a_{-}^{*} \exp (4 R Z)=0 .
\end{aligned}
$$

In the above equations, $a_{+}(Z, T)$ and $a_{-}(Z, T)$ are the normalized slowly varying amplitude envelopes of the symmetric and antisymmetric modes, respectively. $Z$ and $T$ are the normalized propagation distance and time coordinate defined for a frame that moves at the average group velocity of the two modes. The second terms in Eq. (1) account for the effects of IMD, where the normalized parameter $R_{1}$ is a measure of the deviation from the average group delay for the two modes. The third terms represent (anomalous) GVD, which is assumed to be the same for both modes. The fourth and fifth terms represent self-phase modulation and cross-phase modulation (XPM), respectively, and the last nonlinear terms are due to the difference of the phase velocities of the two modes, where $R$ is a measure of the difference of the propagation constants of the two modes. With all the nonlinear terms ignored, Eq. (1) simply describes two supermodes propagating along the fiber independently from each other at different group velocities (with the difference proportional to $R_{1}$ ). When a pulse is launched into only one core, both supermodes are equally excited and eventually separated in time after propagating over a long enough distance, which thus leads to pulse splitting [18]. The value of $R_{1}$ varies over a wide range, depending on the fiber parameters and the pulsewidth, and is negative in normal operating conditions [27]. There exists, however, a specific operating condition for a conventional two-core fiber design at which the value of $R_{1}$ is equal to zero [18].

In practical applications of a two-core fiber, it is customary to directly calculate the pulse envelopes in the individual cores. According to the coupled-mode theory, which assumes weak spatial overlap between the mode fields in the individual cores, the normalized amplitude envelopes of the two supermodes can be expressed as the superposition of the normalized amplitude envelopes of the two modes in the individual cores, denoted as $a_{1}(Z, T)$ and $a_{2}(Z, T)$, respectively [21]

$$
\begin{aligned}
& a_{+}=\frac{1}{2}\left(a_{1}+a_{2}\right) \exp (-i R Z), \\
& a_{-}=\frac{1}{2}\left(a_{1}-a_{2}\right) \exp (i R Z) .
\end{aligned}
$$

By substituting Eq. (2) into Eq. (1), we arrive at the following generalized coupled nonlinear Schrödinger equations:

$$
\begin{aligned}
& i \frac{\partial a_{1}}{\partial Z}+\frac{1}{2} \frac{\partial^{2} a_{1}}{\partial T^{2}}+\left|a_{1}\right|^{2} a_{1}+R a_{2}+i R_{1} \frac{\partial a_{2}}{\partial T}=0 \\
& i \frac{\partial a_{2}}{\partial Z}+\frac{1}{2} \frac{\partial^{2} a_{2}}{\partial T^{2}}+\left|a_{2}\right|^{2} a_{2}+R a_{1}+i R_{1} \frac{\partial a_{1}}{\partial T}=0 .
\end{aligned}
$$

Equation (3) can also be derived directly from the coupledmode theory without going through the conversion from the supermodes [28]. The terms in Eq. (3) that contain $R$ are the conventional linear coupling terms, which account for the periodic power transfer between the two cores in the linear, continuous-wave limit. The modes in the individual cores propagate at the same group velocity (this is expected since the two cores are identical); their group velocity is actually equal to the average group velocity of the two supermodes. The effects of IMD are turned into additional linear coupling between the two cores, i.e., the last terms in Eq. (3) that contain $R_{1}$. These terms are absent in earlier studies [ $\left.\underline{6}-12\right]$ and cannot be eliminated by a transformation of coordinates. Under the coupled-mode theory, the parameter $R_{1}$ is understood as a measure of the wavelength dependence of the coupling coefficient $R$, so it is also referred to as the couplingcoefficient dispersion $[21,28]$. In other words, the IMD in the supermode theory is equivalent to the coupling-coefficient dispersion in the coupled-mode theory; they represent the same physics. Because of the way the parameters are normalized [21,28], $R_{1}$ actually measures the relative significance of the IMD and the GVD in the fiber. We should also note that there are no XPM terms in Eq. (3), which is a result of weak spatial overlap between the mode fields in the two cores, as required by the coupled-mode theory.

In this study, we assume that the pulse is launched into only one core:

$$
a_{1}(0, T)=\sqrt{P_{0}} \operatorname{sech}(T), \quad a_{2}(0, T)=0,
$$

where $P_{0}$ is the normalized peak power. $P_{0}=1$ is the power required for soliton formation in a single-mode fiber, known as the soliton power. To solve Eq. (3), we employ the pseudospectral method in the time domain and the fourth-order Runge-Kutta scheme in the spatial domain with adaptive step-size control.

For the purpose of benchmarking, we first consider a linear fiber, where the nonlinear terms in Eq. (3) are ignored. Figure 1 shows the pulse propagation dynamics for three values of IMD, $R_{1}=0,-0.5$, and -1.0 , where $R=20$. In the absence of IMD, as shown in Fig. 1 (a) for $R_{1}=0$, the pulse is coupled between the two cores periodically and the shortest distance to achieve complete coupling, known as the coupling length, is given by $L_{c}=\pi /(2 R)$ [28]. The pulse becomes broader and broader, as it propagates down the fiber, because of the GVD. In the presence of IMD, the pulse splits and, at the same time, broadens by the GVD. The pulse-splitting effect is characterized by a walk-off distance $L_{w}=1 /\left(2\left|R_{1}\right|\right)$, at which the pulse completely splits into two identical pairs of pulses in the two cores (assuming no pulse broadening) and power exchange between the two cores stops [28]. When the IMD is relatively weak, as shown in Fig. $1(\mathrm{~b})$ for $R_{1}=-0.5$, the pulse significantly broadens before splitting and the combined 

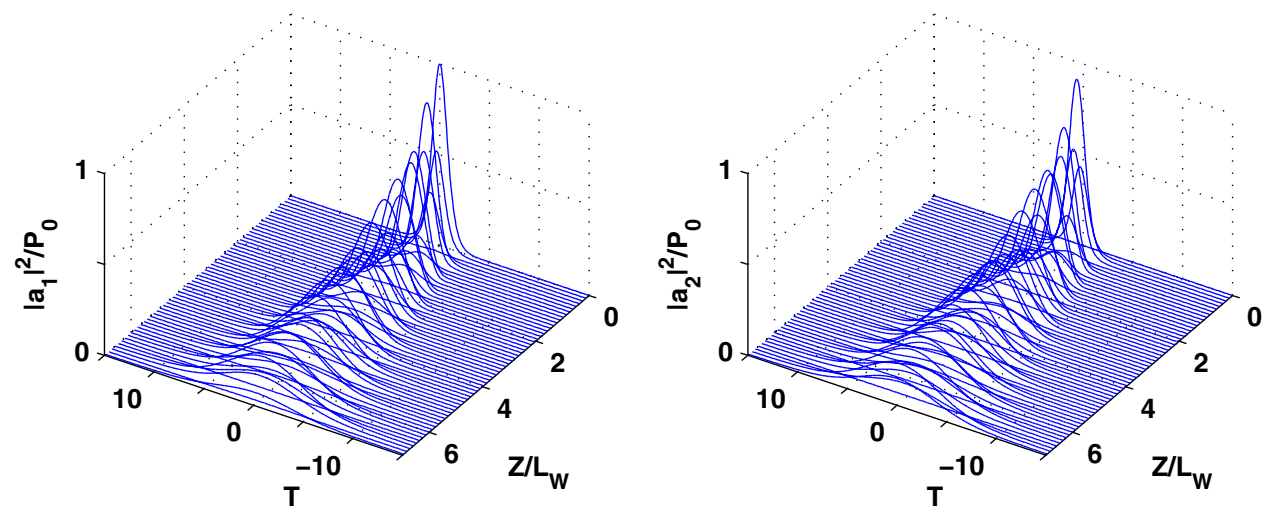

(a)
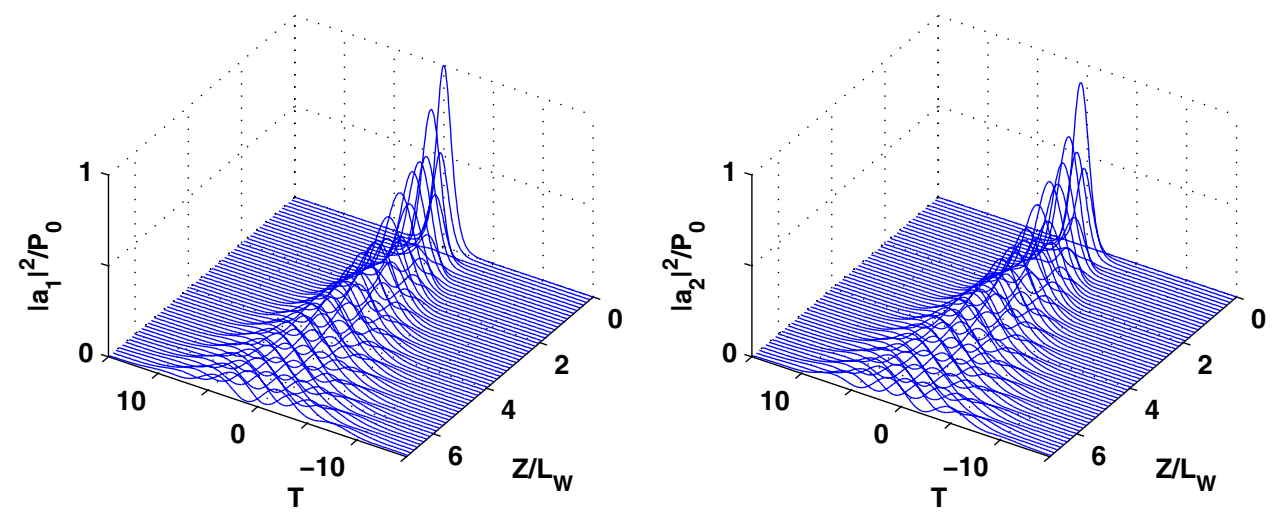

(b)
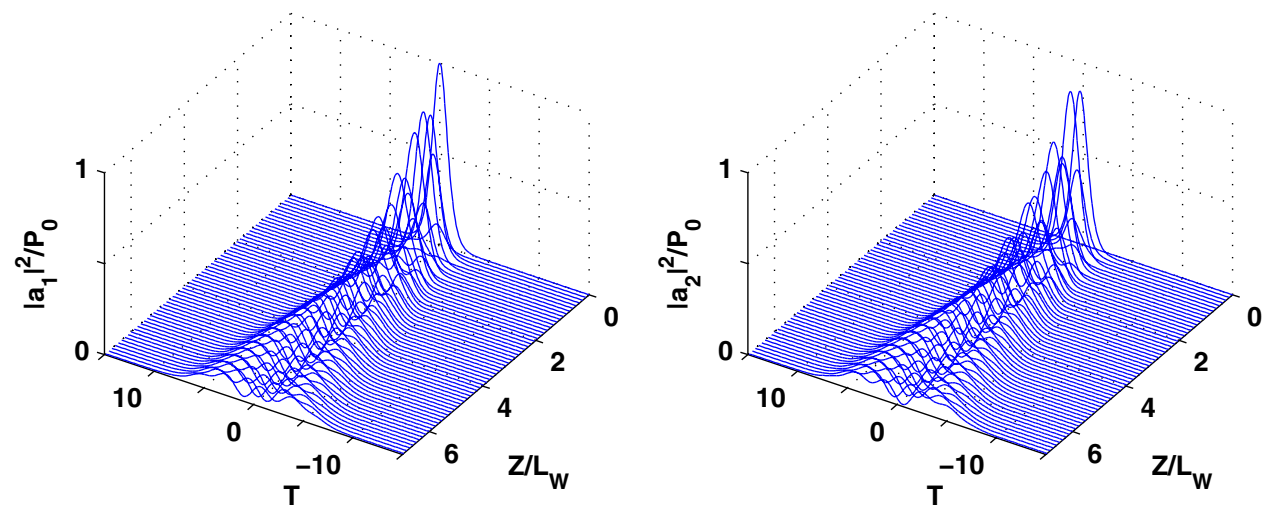

(c)

Fig. 1. (Color online) Pulse propagation in a linear two-core fiber with $R=20$ for (a) $R_{1}=0$, (b) $R_{1}=-0.5$, and (c) $R_{1}=-1.0$, where $L_{w}=1$ in (a) and (b), and $L_{w}=0.5$ in (c).

effects of GVD and IMD result in severe pulse distortion. When the IMD is relatively strong, as shown in Fig. 1(c) for $R_{1}=-1.0$, the pulse splits before the GVD causes significant broadening. These results show that the GVD and the IMD in a two-core fiber produce different effects and both tend to destroy the integrity of the pulse. The question is whether it is possible to balance both GVD and IMD with nonlinearity to keep the integrity of the pulse over a long distance.

The above examples represent the typical situations where the walk-off length is much longer than the coupling length. From the definitions of the normalized parameters [21,28], $R$ and $R_{1}$ can be expressed in terms of the dispersion length of the fiber $L_{D}$ as $R=(\pi / 2)\left(L_{D} / l_{c}\right)$ and $\left|R_{1}\right|=0.5\left(L_{D} / l_{w}\right)$, where $l_{c}$ and $l_{w}$ are the actual (nonnormalized) coupling length and walk-off length of the fiber, respectively. Consequently, $R \gg$ 1 implies that the coupling length is much shorter than the dispersion length. In the case $R_{1}=-0.5$, the walk-off length is equal to the dispersion length, while in the case $R_{1}=-1.0$, the walk-off length becomes shorter than the dispersion length.

\section{SUPPRESSION OF PULSE SPLITTING WITH NONLINEARITY}

Early analyses of nonlinear two-core fibers [6-10], which overlook IMD, confirm the possibility of distortion-free propagation 

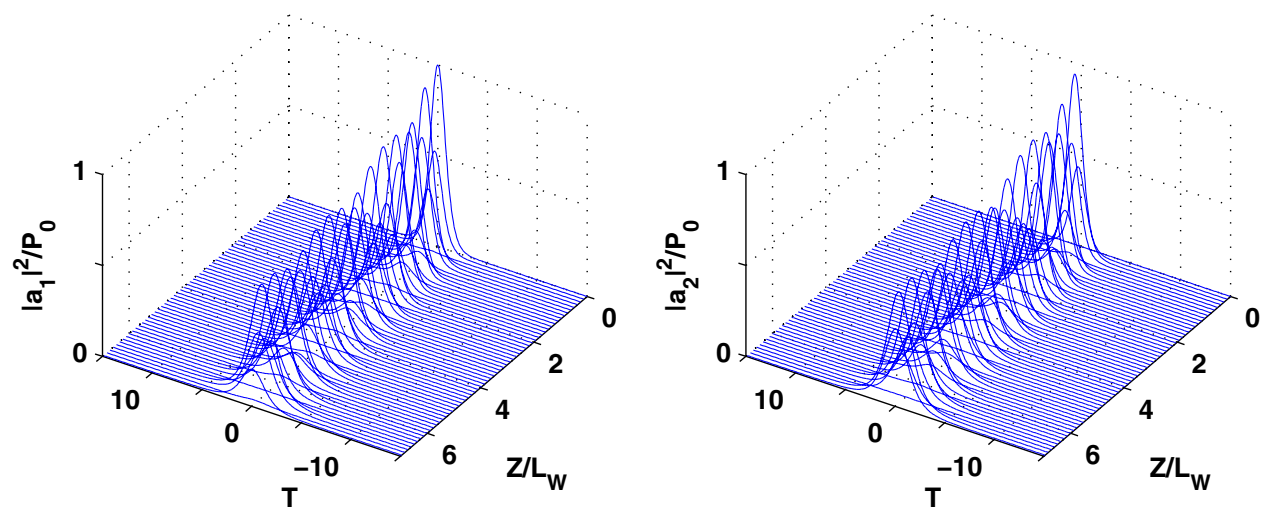

(a)
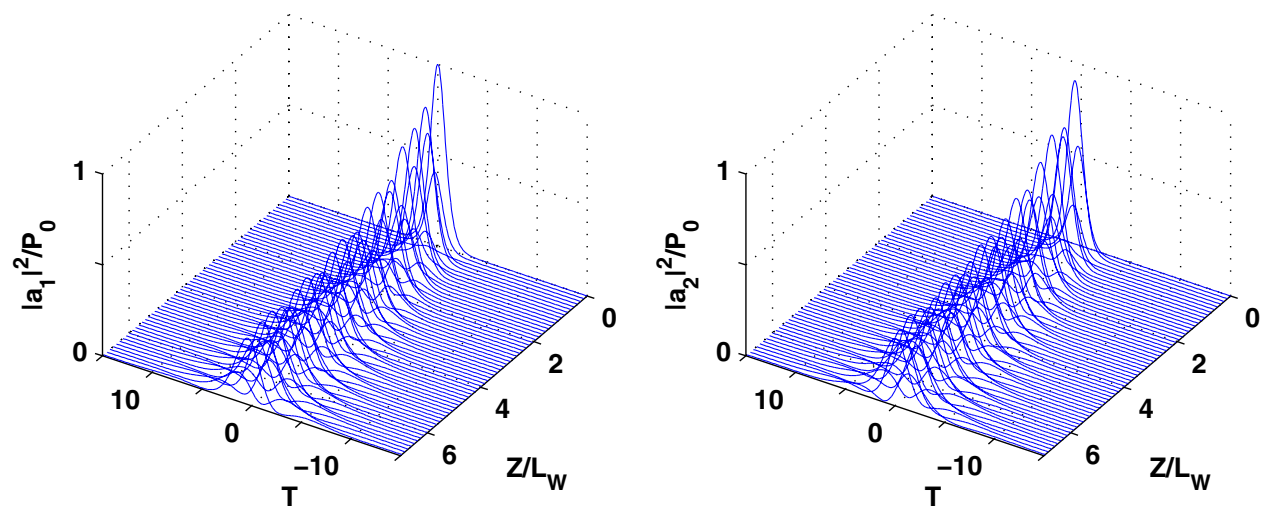

(b)
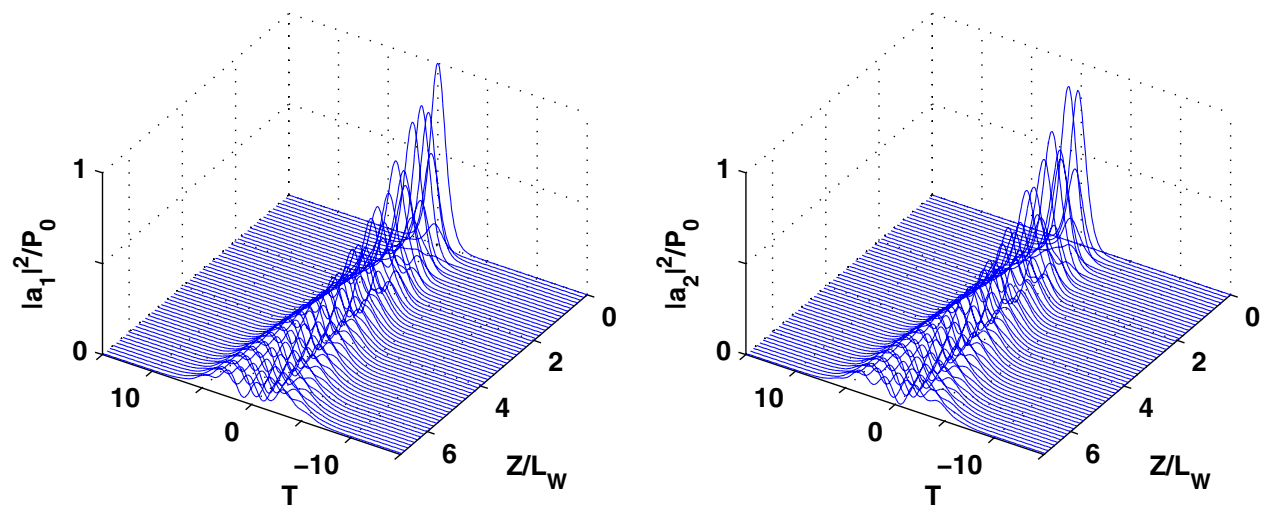

(c)

Fig. 2. (Color online) Pulse propagation in a nonlinear two-core fiber with $R=20$ at $P_{0}=1$ for (a) $R_{1}=0$, (b) $R_{1}=-0.3$, and (c) $R_{1}=-1.0$, where $L_{w}=1$ in (a) and $L_{w}=1 /\left(2\left|R_{1}\right|\right)$ in (b) and (c).

at $P_{0}=1$, as shown in Fig. 2(a) for $R_{1}=0$ (where $R=20$ ). With $P_{0}=1$, the pulse-broadening effect due to GVD alone is completely balanced by nonlinearity, as we can see by comparing Figs. 1(a) and 2(a). When the effects of IMD are included in the analysis, however, the input pulse with $P_{0}=1$ can no longer propagate without distortion. Even if the IMD is weak, pulse distortion can be significant, as shown in Fig. 2(b) for $R_{1}=-0.3$. When the IMD is sufficiently strong, pulse splitting becomes obvious, as shown in Fig. $2(\mathrm{c})$ for $R_{1}=-1.0$. A comparison of Figs. 1(c) and 2(c) confirms that, in the presence of strong IMD, soliton pulses split like low-power pulses.
While $P_{0}=1$ is insufficient to suppress the effects of IMD, we increase the input power to $P_{0}=2$. Figures $3(\mathrm{a})-3(\mathrm{~d})$ show the propagation dynamics at $P_{0}=2$ for four values of IMD, $R_{1}=-0.3,-0.5,-0.9$, and -1.2 , where $R=20$. We find that the integrity of the input pulse can be maintained when $\left|R_{1}\right|$ is not larger than $\sim 0.5$. The pulse starts to deteriorate at a larger value of $\left|R_{1}\right|$ and clearly splits at $R_{1}=-1.2$. These results suggest the possibility of suppressing the effects of IMD with nonlinearity, provided that $\left|R_{1}\right|$ is not larger than a certain value. For $P_{0}=2$, the maximum value of $\left|R_{1}\right|$ that can be balanced by nonlinearity is $\sim 0.5$. 


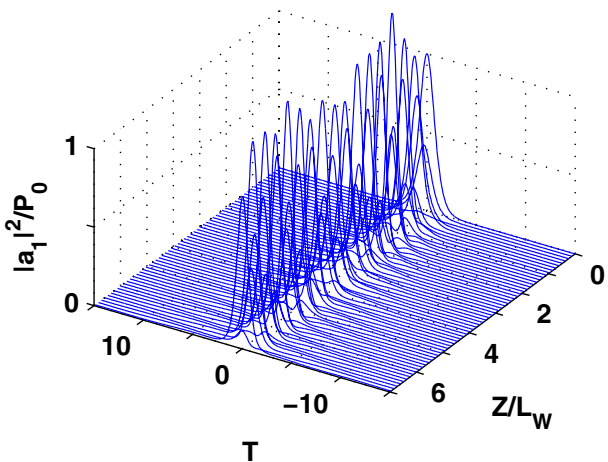

(a)

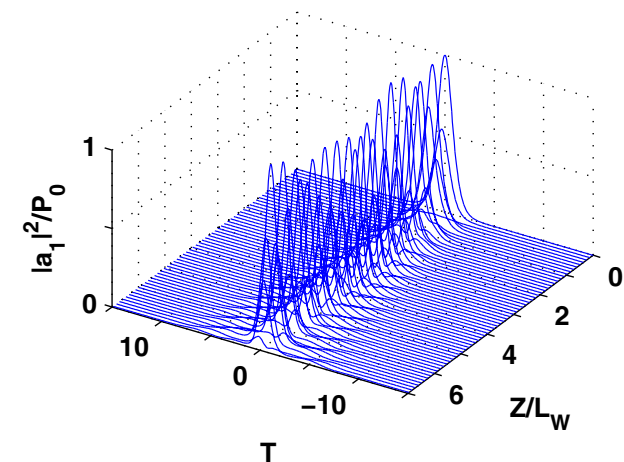

(b)

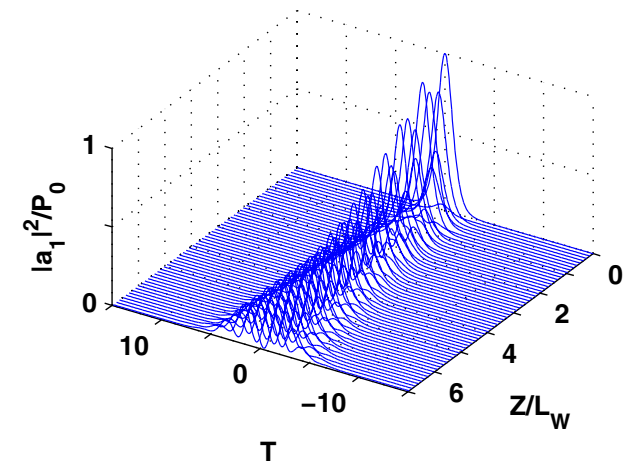

(c)
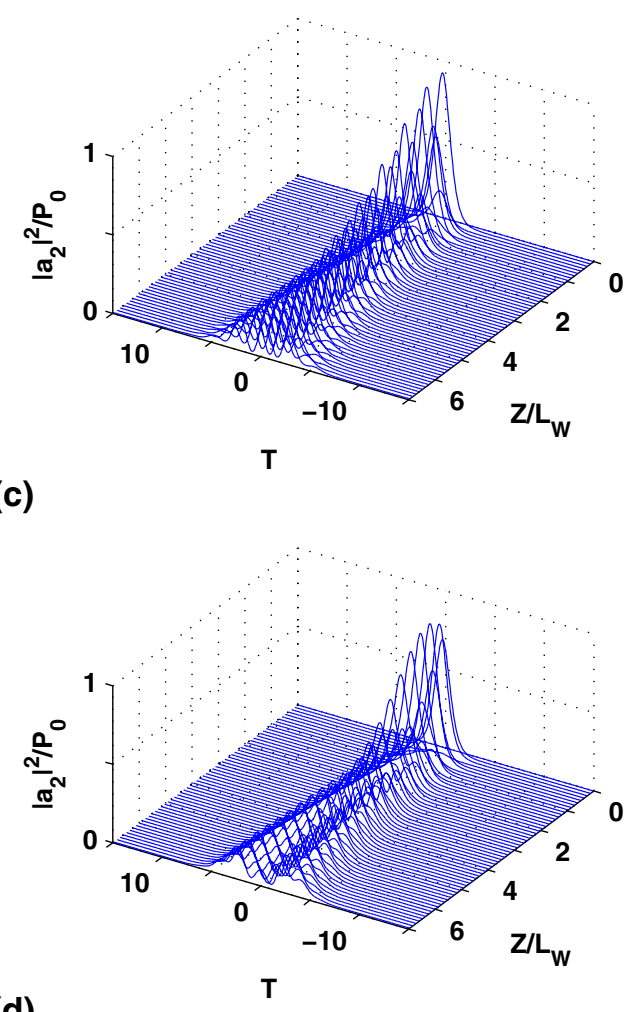

(d)
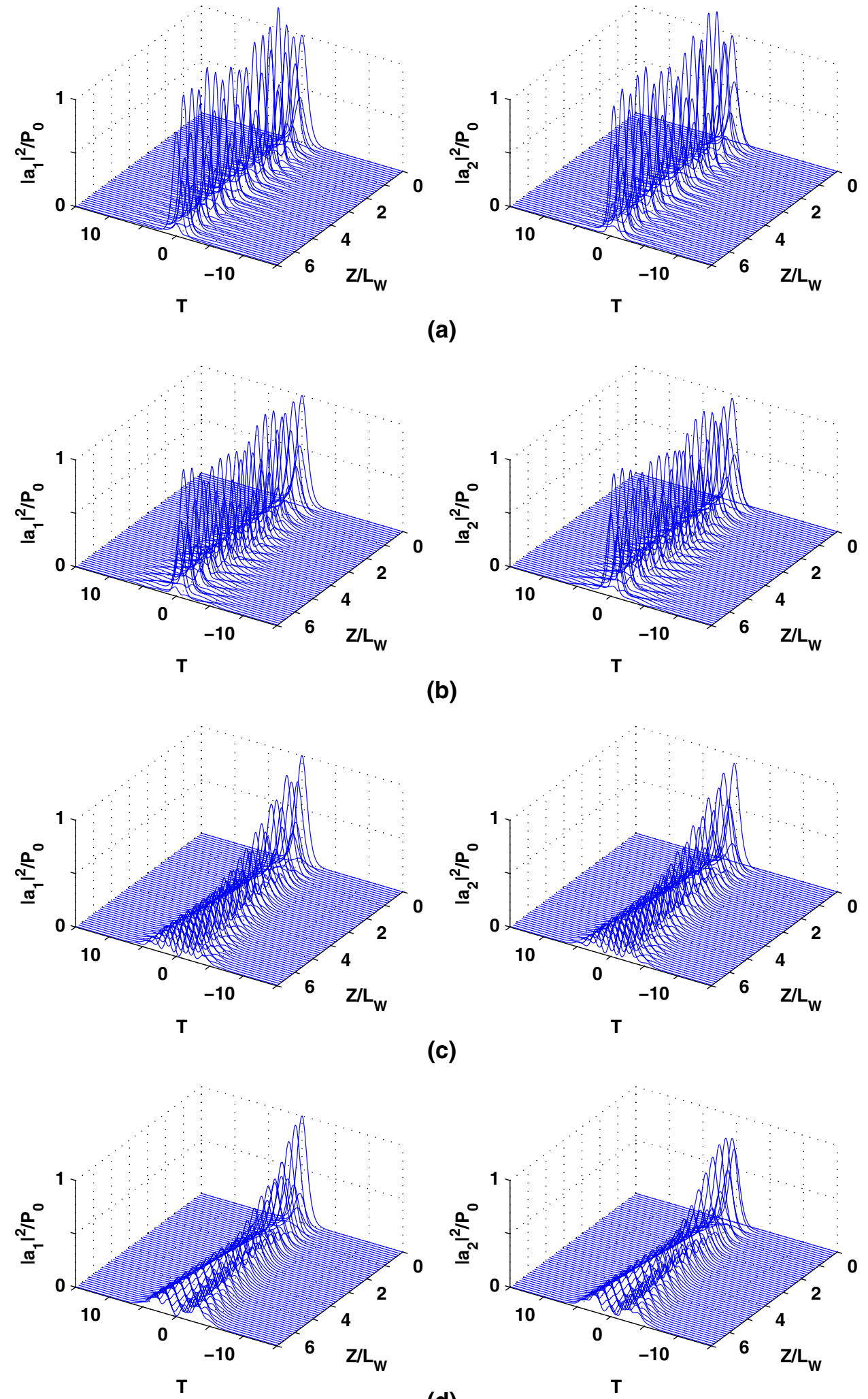

a)

Fig. 3. (Color online) Pulse propagation in a nonlinear two-core fiber with $R=20$ at $P_{0}=2$ for (a) $R_{1}=-0.3$, (b) $R_{1}=-0.5$, (c) $R_{1}=-0.9$, and (d) $R_{1}=-1.2$, where $L_{w}=1 /\left(2\left|R_{1}\right|\right)$.

We continue to increase the input power and find that the maximum value of $\left|R_{1}\right|$ that can be balanced by nonlinearity increases from $\sim 0.5$ to $\sim 0.9$, as the input power $P_{0}$ increases from 2 to 5 . Figures $\underline{4(a)}-\underline{4(d)}$ show the propagation dynamics at $P_{0}=4$ for four values of IMD, $R_{1}=-0.5,-0.7,-1.0$, and -2.0 , where $R=20$. At $P_{0}=4$, the pulse maintains its integrity up to $\left|R_{1}\right|=\sim 0.7$ and becomes significantly distorted above this value of $\left|R_{1}\right|$. As the IMD becomes strong enough, the 

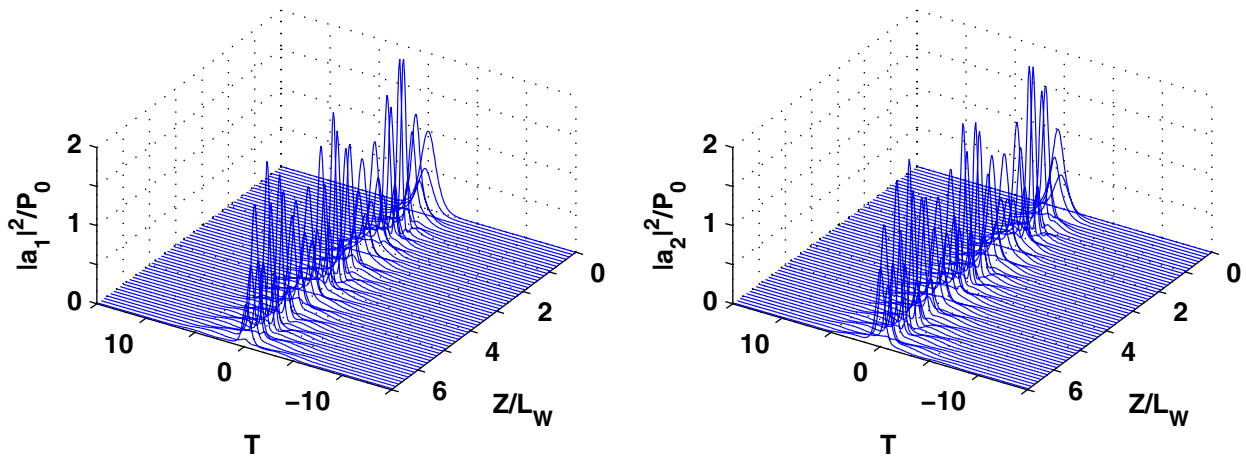

(a)
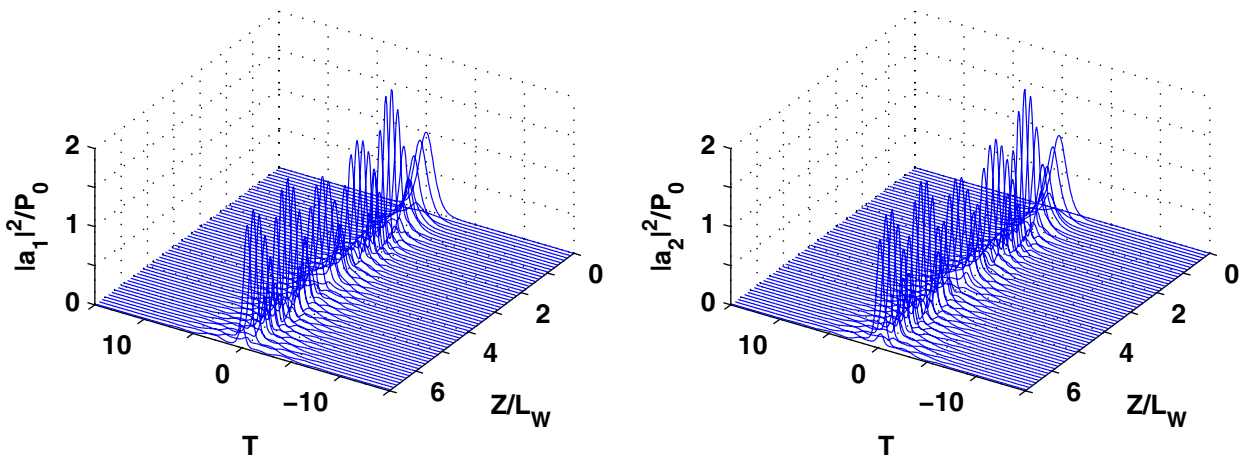

(b)
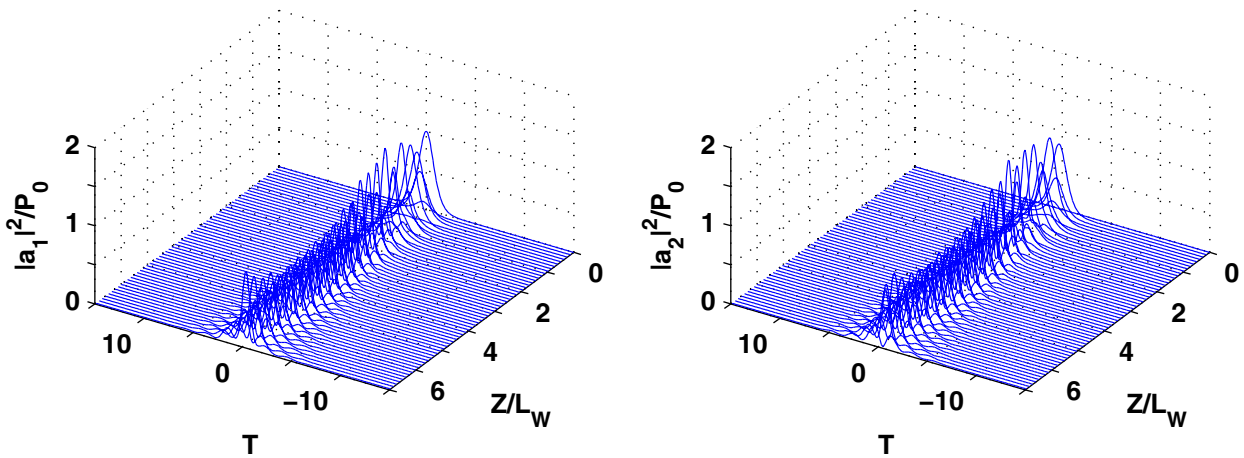

(c)
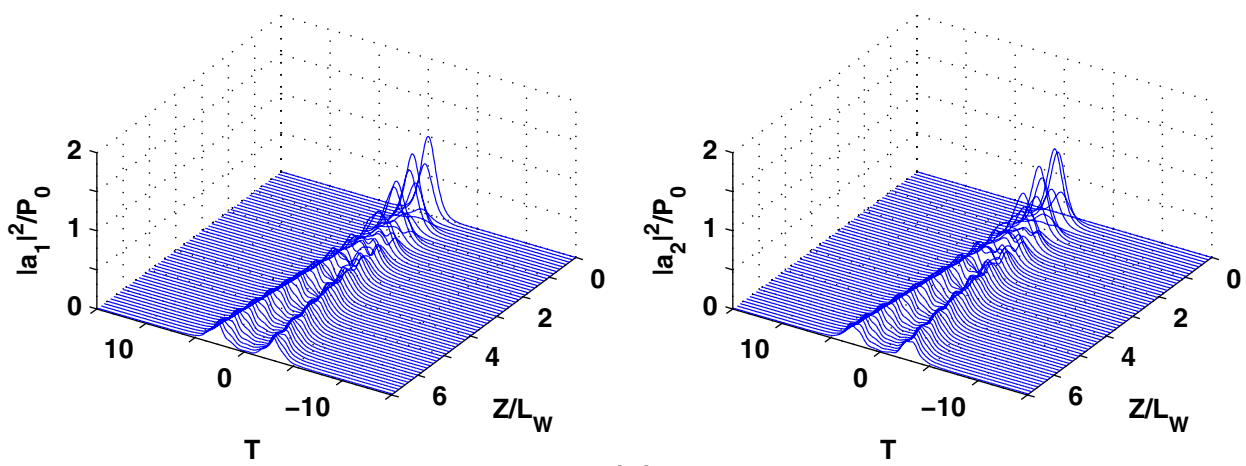

(d)

Fig. 4. (Color online) Pulse propagation in a nonlinear two-core fiber with $R=20$ at $P_{0}=4$ for (a) $R_{1}=-0.5$, (b) $R_{1}=-0.7$, (c) $R_{1}=-1.0$, and (d) $R_{1}=-2.0$, where $L_{w}=1 /\left(2\left|R_{1}\right|\right)$.

input pulse eventually splits into four identical pulses, as shown in Fig. 4(d). Since each of the split pulses carries a quarter of the input power (i.e., the soliton power), these pulses are actually soliton pulses, which explains why they do not suffer from further broadening by the GVD as they propagate down the fiber.

We also find that, for the nonlinearity to balance the GVD and the IMD, the value of the linear coupling coefficient 

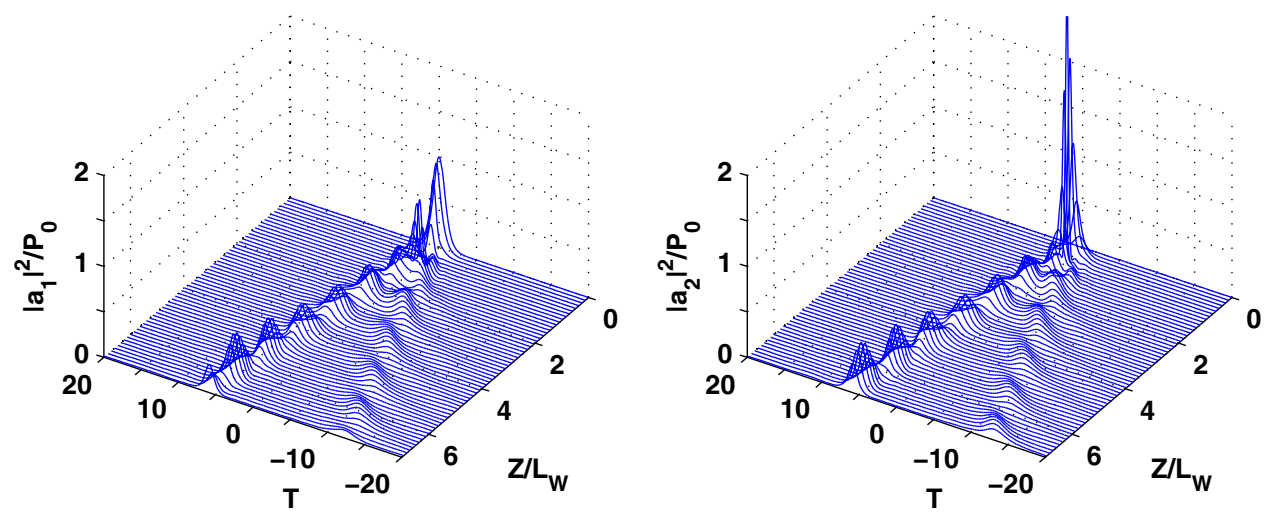

(a)
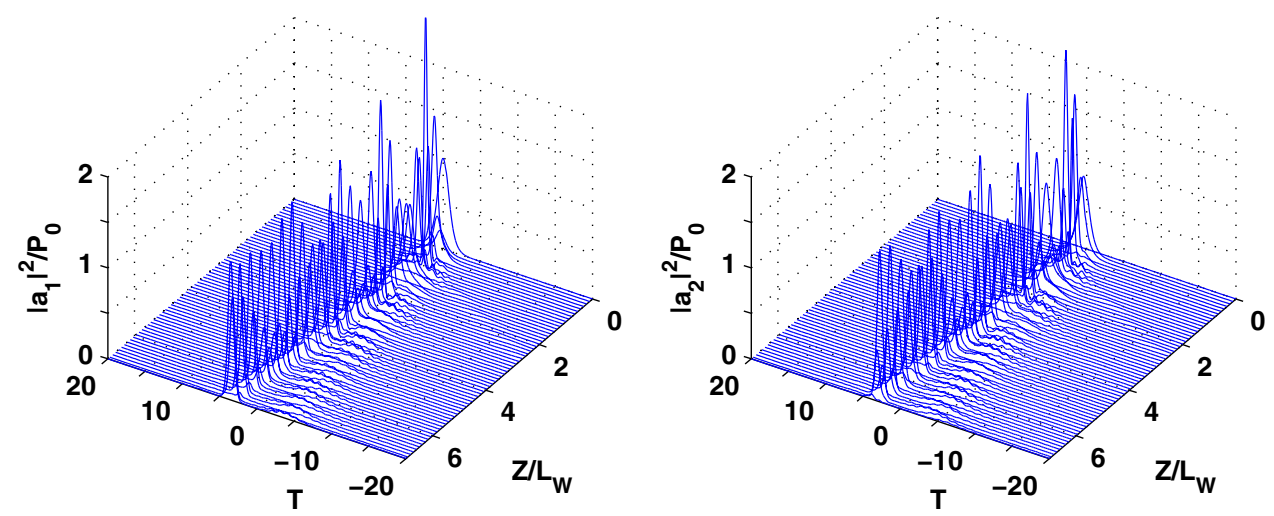

(b)
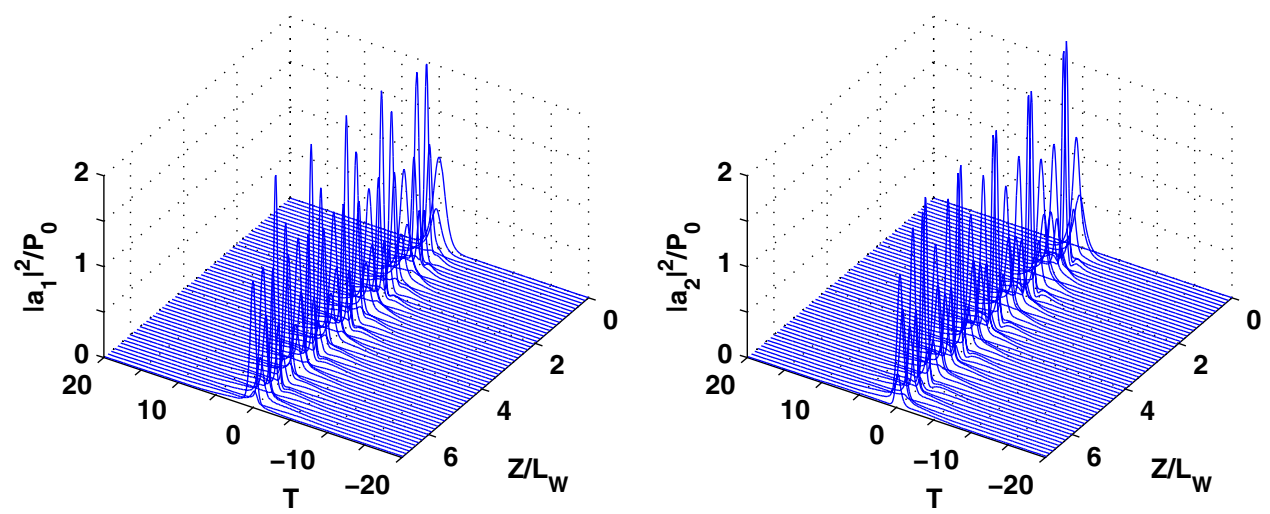

(c)

Fig. 5. (Color online) Pulse propagation in a nonlinear two-core fiber with $R_{1}=-0.3$ at $P_{0}=4$ for (a) $R=2$, (b) $R=6$, and (c) $R=20$, where $L_{w}=1 /\left(2\left|R_{1}\right|\right)=1.67$.

$R$ must be much larger than 1 ( $R \gg 1$ ), or the coupling length must be much shorter than the walk-off length $\left(L_{c} \ll L_{w}\right)$. This condition is satisfied in the examples given in Figs. 2-4, where $R=20$. Figures $5(\mathrm{a})-5(\mathrm{c})$ show the propagation dynamics at $P_{0}=4$ for a fiber with $R_{1}=-0.3$ at $R=2,6$, and 20, respectively. When $R$ is small, as shown in Figs. 5(a) and $5(\mathrm{~b})$, the input pulse splits quickly into two pulses with unequal amplitudes. When $R$ is large, no such splitting takes place and the input pulse maintains its integrity, as shown in Fig. 5(c).

Our numerical results also suggest the existence of a threshold input power for the suppression of pulse splitting at a given value of $R_{1}$. For example, the threshold input powers for $R_{1}=-0.5$ and -0.7 are $P_{0} \sim 2$ and 4 , respectively. The analytical formula for the estimation of the threshold power to overcome the IMD in a multimode fiber [23], which assumes a predominant IMD over GVD, is not applicable to our situation where IMD is relatively weak. We can make a more relevant comparison with the study of a nonlinear birefringent fiber [26], where coupled equations similar to Eq. (1) are solved numerically for soliton-like pulse propagation. Assuming both polarized modes are equally excited, the threshold input power required for compensating the polarization-mode dispersion with a normalized value of 1.0 
(which plays a role similar to $R_{1}$ in a two-core fiber) is $P_{0} \sim 4$ which is comparable to our finding.

We have limited our study to a relatively low input power (up to several times of the soliton power) to justify the omission of high-order nonlinear effects in our equations. To compensate for stronger IMD, it is necessary to use higher input power, which, however, might dictate the inclusion of other nonlinear effects. The propagation dynamics at higher power are expected to be more complicated and will need further investigation.

\section{CONCLUSION}

It is possible to prevent a pulse launched into a two-core fiber from broadening and splitting by balancing the (anomalous) GVD and the IMD with the Kerr nonlinearity in the fiber. The peak power of the pulse required must be larger than the soliton power. There exists a maximum amount of IMD that can be balanced (generally $\left|R_{1}\right|<1$ for an input peak power up to a few times of the soliton power), which increases with the peak power. The linear coupling between the two cores must also be sufficiently strong $(R \gg 1)$. These conditions correspond to the situation of launching a pulse with a width of $0.1-1 \mathrm{ps}$ at a center wavelength of $\sim 1.5 \mu \mathrm{m}$ into a conventional two-core silica fiber that has a core-to-core separation 6-10 times that of the core radius [27]. For a conventional two-core fiber, the relationship between $R_{1}$ and $R$ is very much fixed $[18,27]$ and the nonlinearity available is similar to that of a conventional single-mode fiber. On the other hand, photoniccrystal two-core fibers could offer much stronger nonlinearity and allow more flexible choices of the values of $R_{1}$ and $R$, and, therefore, should provide a convenient platform for experimental demonstration of the effects discussed in this study.

\section{ACKNOWLEDGMENTS}

This research is partially supported by the University of Hong Kong Incentive Award Scheme.

\section{REFERENCES}

1. A. Hasegawa and F. Tappert, "Transmission of stationary nonlinear optical pulses in dispersive dielectric fibers. I. Anomalous dispersion," Appl. Phys. Lett. 23, 142-144 (1973).

2. L. F. Mollenauer, R. H. Stolen, and J. P. Gordon, "Experimental observation of picosecond pulse narrowing and solitons in optical fibers," Phys. Rev. Lett. 45, 1095-1098 (1980).

3. J. R. Taylor, ed., Optical Solitons: Theory and Experiments (Cambridge University, 1992).

4. N. Akhmediev and A. Ankiewicz, Solitons: Nonlinear Pulses and Beams (Chapman \& Hall, 1997).

5. Y. S. Kivshar and G. P. Agrawal, Optical Solitons: From Fibers to Photonic Crystals (Academic, 2003).

6. S. Trillo, S. Wabnitz, E. M. Wright, and G. I. Stegeman, "Soliton switching in fiber nonlinear directional couplers," Opt. Lett. 13, 672-674 (1988).

7. J. M. Soto-Crespo and E. M. Wright, "All-optical switching of solitons in two- and three-core nonlinear fiber couplers," J. Appl. Phys. 70, 7240-7243 (1991).
8. P. L. Chu, B. A. Malomed, and G. D. Peng, "Analytical solution to soliton switching in nonlinear twin-core fibers," Opt. Lett. 18, 328-330 (1993).

9. Y. S. Kivshar, "Switching dynamics of solitons in fiber directional couplers," Opt. Lett. 18, 7-9 (1993).

10. N. Akhmediev and J. M. Soto-Crespo, "Propagation dynamics of ultra-short pulses in nonlinear fiber couplers," Phys. Rev. E 49, 4519-4529 (1994).

11. S. R. Friberg, Y. Silberberg, M. K. Oliver, M. J. Andrejco, M. A. Saifi, and P. W. Smith, "Ultrafast all-optical switching in a dualcore fiber nonlinear coupler," Appl. Phys. Lett. 51, 1135-1137 (1987).

12. S. R. Friberg, A. M. Weiner, Y. Silberberg, B. G. Sfez, and P. W. Smith, "Femtosecond switching in a dual-core-fiber nonlinear coupler," Opt. Lett. 13, 904-906 (1988).

13. B. Zhu, T. F. Taunay, M. F. Yan, J. M. Fini, M. Fishteyn, E. M. Monberg, and F. V. Dimarcello, "Seven-core multicore fiber transmissions for passive optical network," Opt. Express 18, 11117-11122 (2010).

14. K. Mukasa, K. Imamura, Y. Tsuchida, and R. Sugizaki, "Multicore fibers for large capacity SDM," in Proceedings of Optical Fiber Communication Conference, OSA Technical Digest Series (Optical Society of America, 2011), p. OWJ1.

15. J. Sakaguchi, Y. Awaji, N. Wada, T. Hayashi, T. Nagashima, T. Kobayashi, and M. Watanabe, "Propagation characteristics of seven-core fiber for spatial and wavelength division multiplexed 10 Gbit/s channels," in Proceedings of Optical Fiber Communication Conference, OSA Technical Digest Series (Optical Society of America, 2011), p. OWJ2.

16. A. Betlej, S. Suntsov, K. G. Makris, L. Jankovic, D. N. Christodoulides, G. I. Stegeman, J. Fini, R. T. Bise, and D. J. Digiovanni, "All-optical switching and multifrequency generation in a dual-core photonic crystal fiber," Opt. Lett. 31, 1480-1482 (2006).

17. P. Li, J. L. Zhao, and X. J. Zhang, "Nonlinear coupling in triangular triple-core photonic crystal fibers," Opt. Express 18, 26828-26833 (2010).

18. K. S. Chiang, "Intermodal dispersion in two-core optical fibers," Opt. Lett. 20, 997-999 (1995).

19. K. S. Chiang, Y. T. Chow, D. J. Richardson, D. Traverner, L. Dong, L. Reekie, and K. M. Lo, "Experimental demonstration of intermodal dispersion in a two-core optical fiber," Opt. Commun. 143, 189-192 (1997).

20. P. Peterka, P. Honzatko, J. Kanka, V. Matejec, and I. Kasik, "Generation of high-repetition rate pulse trains in a fiber laser through a twin-core fiber," Proc. SPIE 5036, 376-381 (2003).

21. K. S. Chiang, "Propagation of short optical pulses in directional couplers with Kerr nonlinearity," J. Opt. Soc. Am. B 14, 1437-1443 (1997).

22. M. Liu and K. S. Chiang, "Nonlinear switching of ultrashort pulses in multicore fibers," IEEE J. Quantum Electron. 47, 1499-1505 (2011).

23. A. Hasegawa, "Self-confinement of multimode optical pulse in a glass fiber," Opt. Lett. 5, 416-418 (1980).

24. B. Crosignani and P. Di Porto, "Soliton propagation in multimode optical fibers," Opt. Lett. 6, 329-331 (1981).

25. B. Crosignani, A. Cutolo, and P. Di Porto, "Coupled-mode theory of nonlinear propagation in multimode and single-mode fibers: envelope solitons and self-confinement," J. Opt. Soc. Am. 72, 1136-1141 (1982).

26. C. R. Menyuk, "Stability of solitons in birefringent optical fibers II. Arbitrary amplitudes,” J. Opt. Soc. Am. B 5, 392-402 (1988).

27. P. Shum, K. S. Chiang, and W. A. Gambling, "Switching dynamics of short optical pulses in a nonlinear directional coupler," IEEE J. Quantum Electron. 35, 79-83 (1999).

28. K. S. Chiang, "Coupled-mode equations for pulse switching in parallel waveguides," IEEE J. Quantum Electron. 33, 950-954 (1997). 